Purdue University Purdue e-Pubs

9-1-2007

\title{
Theory of high bias coulomb blockade in ultrashort molecules
}

Bhaskaran Muralidharan

Purdue University, bmuralid@purdue.edu

Avik W. Ghosh

Swapan K. Pati

Supriyo Datta

Birck Nanotechnology Center and Purdue University, datta@purdue.edu

Follow this and additional works at: http://docs.lib.purdue.edu/nanodocs

Muralidharan, Bhaskaran; Ghosh, Avik W.; Pati, Swapan K.; and Datta, Supriyo, "Theory of high bias coulomb blockade in ultrashort molecules" (2007). Other Nanotechnology Publications. Paper 12.

http://docs.lib.purdue.edu/nanodocs/12

This document has been made available through Purdue e-Pubs, a service of the Purdue University Libraries. Please contact epubs@purdue.edu for additional information. 


\title{
Theory of High Bias Coulomb Blockade in Ultrashort Molecules
}

\author{
Bhaskaran Muralidharan, Avik W. Ghosh, Swapan K. Pati, and Supriyo Datta, Fellow, IEEE
}

\begin{abstract}
We point out that single electron charging effects such as coulomb blockade (CB) and high-bias staircases play a crucial role in transport through single ultrashort molecules. A treatment of CB through a prototypical molecule, benzene, is developed using a master-equation in its complete many-electron Fock space, evaluated through exact diagonalization or full configuration interaction (CI). This approach can explain a whole class of nontrivial experimental features including vanishing zero bias conductances, sharp current onsets followed by ohmic current rises, and gateable current levels and conductance structures, most of which cannot be captured even qualitatively within the traditional self-consistent field (SCF) approach coupled with perturbative transport theories. By comparing the two approaches, namely SCF and CB, in the limit of weak coupling to the electrode, we establish that the inclusion of strong correlations within the molecule becomes critical in addressing the above experiments. Our approach includes on-bridge correlations fully, and is therefore well-suited for describing transport through short molecules in the limit of weak coupling to electrodes.
\end{abstract}

Index Terms-Configuration interaction, Coulomb Blockade, molecular conduction, nonequilibrium Green's function, self-consistent field.

\section{INTRODUCTION}

$\mathbf{T}$ HEORETICAL calculations on single molecule conduction have typically employed coherent nonequilibrium Green's function (NEGF) theories ("Landauer limit") [1], [2] coupled with self-consistent fields (SCF) to describe charging effects. Though fairly successful in describing many aspects of single molecule conduction [3]-[7], there have been important discrepancies between theory and experiment [8]. The most common ones include poor match between theoretical and experimental current levels and zero-bias currents [3], [4], [8]. It was also pointed out in [9] that a whole class of experimental $I-V$ 's show features, which cannot be captured even qualitatively using an SCF theory. Charging energies of short molecules ( $\sim 3 \mathrm{eV}$ for benzene) are often larger than their electrode coupling $(<0.2 \mathrm{eV}$ for benzene di-thiol on gold), and thus could be in the coulomb blockade $(\mathrm{CB})$ regime where

\footnotetext{
Manuscript received August 31, 2006; revised January 9, 2007. This work was supported in part by NCN and in part by DARPA-AFOSR. The reveiw of this paper was arranged by Associate Editor R. Lake.

B. Muralidharan and S. Datta are with the School of Electrical and Computer Engineering and Network for Computational Nanotechnology, Purdue University, West Lafayette, IN 47907 USA (e-mail: bmuralid@ecn.purdue.edu).

A. W. Ghosh is with the School of Electrical and Computer Engineering, University of Virginia, Charlottesville, VA 22904 USA.

S. K. Pati is with the Theoretical Sciences Unit and Chemistry and Physics of Materials Unit, Jawaharlal Nehru Center for Advanced Scientific Research, Jakkur Campus, Bangalore 560064, India.

Color versions of one or more of the figures in this paper are available online at http://ieeexplore.ieee.org.

Digital Object Identifier 10.1109/TNANO.2007.898605
}

single-electron charging effects could dominate. It is thus debatable whether it is better described as a quantum wire in the SCF regime, or as a quantum dot array in the $\mathrm{CB}$ regime. Nevertheless the wisdom of SCF approaches must be scrutinized especially for conduction through shorter molecules. The purpose of this paper is to present a CB approach to molecular conduction using a benzene molecule as prototype, and establish it as a different viewpoint from the conventional NEGF-SCF treatment. Furthermore features obtained via the CB approach can semiquantitatively explain several nontrivial features commonly observed [10]-[14] in experiments.

It is common to distinguish between two regimes of transport: a) an SCF regime where the dominant energy scale is the contact coupling, allowing for fractional charge transfer through the system; and b) a CB regime where the dominant energy scale is the single-electron charging, leading to integral charge transfer. In the SCF regime the description of transport via noninteracting single-particle energy levels can be justified. In this limit, it is common to use the SCF-NEGF scheme that takes charging effects into account. Here the molecular Hamiltonian is described by a set of single-particle levels, which are coupled to reservoirs through their self-energies. The electron interactions are taken into account using SCF schemes as shown in the block diagram in Fig. 1(a). All quantities in the NEGF formalism are matrices of dimension $N \times N, N$ being the number of single-particle basis functions used. This allows for an accurate description of quantum chemistry of both the isolated molecule and its bonding to the contacts [15]. In the CB regime with weak contact coupling, charging effects dominate, and the use of single-particle basis sets may be questionable. In such cases, it may be preferential to employ a multielectron description where feasible. A single spin degenerate energy level, for example shown in Fig. 1(b), is a set of four levels in its multielectron Fock space. The inclusion of all such configurations in the multielectron Fock space is the central idea behind a full configuration interaction (CI) approach. The central quantities in this CI method are now matrices of dimension $2^{N} \times 2^{N}$, thereby accounting for strong interaction accurately. Often, one may also use a partial set of the many-electron spectrum, of specific relevance to the problem, leading to a partial CI expansion [16]. The weakly coupled contacts are treated perturbatively using transition rates between states differing by a single electron [5]. It is interesting to note that most theoretical efforts in molecular conduction have been in the ballistic SCF regime, in which relaxation effects due to electron-phonon interaction are not taken into account. Our paper is thus a concrete attempt towards CI based transport in the sequential tunneling regime [19], [40] and neglects effects of electron-phonon interactions. These interactions could technically be included within the same framework [17], [18] as already noted. 
(a)
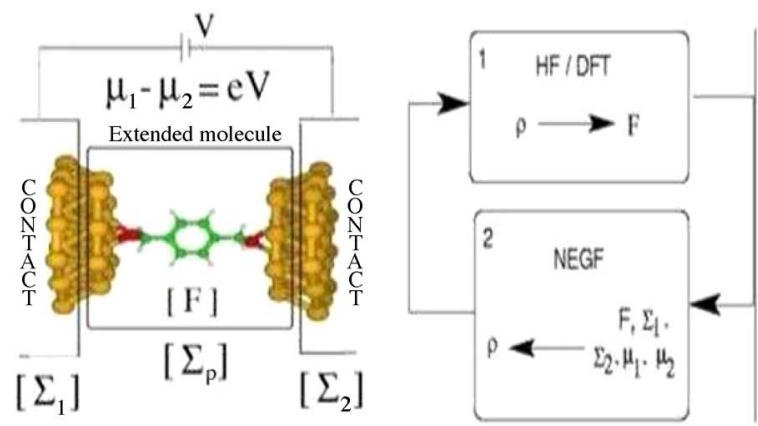

(b)

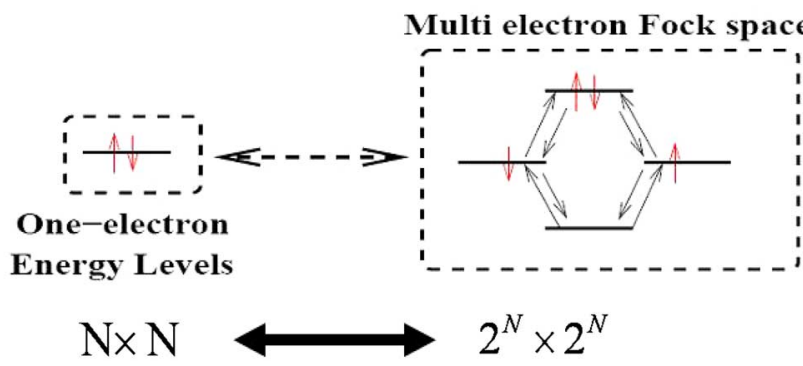

Fig. 1. Our system is a benzene molecule coupled to metallic contacts. Single molecule transport calculations typically employ the NEGF-SCF prescription. The Block diagram depicts the basic scheme. While quantities such as the Hamiltonian are in the one-electron space of dimensions $N \times N, N$ being the number of basis functions. Our CB description involves the full many electron Fock space of dimensions $2^{N} \times 2^{N}$ as shown in (b) using a single spin-degenerate level as an example. The use of full many-electron space captures the correlations exactly within the framework of the given $N \times N$ one-electron Hamiltonian, and is often referred to as a full CI approach.

This paper is organized as follows: we begin by defining an appropriate many-body Hamiltonian for benzene whose parameters are benchmarked based on well-established mean-field techniques. We then illustrate how a CB treatment is conceptually different from the standard SCF treatment in the weak coupling limit, not only under nonequilibrium conditions, but even under equilibrium conditions. We then point out the importance of inclusion of excited states in transport, that naturally arise within our CI approach. The progressive access of these excited states leads to transport signatures under various nonequilibrium conditions. Before we conclude, a few $\mathrm{CB}$ fits to experimental data are presented in support of our analysis.

\section{The Model Hamiltonian ANd EQuilibrium Properties}

An appropriate model Hamiltonian is usually described with an adequate basis set. In this paper, we use a tight binding Hamiltonian with one $p_{z}$ orbital per site to describe our CI based scheme. Although this generates just a minimal $6 \times$ six single-particle basis set, its many-electron space is $2^{12} \times 2^{12}$ in size. Besides, our objective here is to describe the $\mathrm{CI}$ approach for transport and compare it with the SCF approach for the same Hamiltonian. Better quantum chemical descriptions within the CI approach can be achieved by starting with a reduced but more accurate one-particle Hamiltonian, but we leave these for future work.
One begins with the model Hamiltonian in second quantized notation

$$
\begin{aligned}
\hat{H}=\sum_{\alpha} \epsilon_{\alpha} n_{\alpha}+\sum_{\alpha \neq \beta} t_{\alpha \beta} c_{\alpha}^{\dagger} c_{\beta}+\sum_{\alpha, \sigma} U_{\alpha \alpha} n_{\alpha \sigma} n_{\alpha \bar{\sigma}} \\
+\frac{1}{2} \sum_{\alpha \neq \beta} U_{\alpha \beta} n_{\alpha} n_{\beta}
\end{aligned}
$$

where $\alpha, \beta$ correspond to the orbital indexes of the frozen $p_{z}$ orbitals for carbon sites on the benzene ring,and $\sigma, \bar{\sigma}$ represent a particular spin and its reverse. In connection to its equilibrium configuration, it is more convenient to work with onsite energies $\tilde{\epsilon}$ defined as

$$
\tilde{\epsilon}_{\alpha}=\epsilon_{\alpha}+U_{\alpha \alpha}\left\langle n_{\alpha \bar{\sigma}}\right\rangle+\frac{1}{2} \sum_{\alpha \neq \beta} U_{\alpha \beta}\left\langle n_{\beta}\right\rangle
$$

where $\tilde{\epsilon}_{\alpha}$ 's denote the mean-field on-site energies in the equilibrium charge neutral configuration of the molecule and $\langle n\rangle$ represents its mean-field value. Now the model Hamiltonian is simply rewritten as

$$
\begin{aligned}
\hat{H} & =\sum_{\alpha} \tilde{\epsilon}_{\alpha} n_{\alpha}+\sum_{\alpha \neq \beta} t_{\alpha \beta} c_{\alpha}^{\dagger} c_{\beta}+\sum_{\alpha, \sigma} U_{\alpha \alpha}\left(n_{\alpha \sigma}-\left\langle n_{\alpha \sigma}\right\rangle\right) \\
& \times\left(n_{\alpha \bar{\sigma}}-\left\langle n_{\alpha \bar{\sigma}}\right\rangle\right)+\frac{1}{2} \sum_{\alpha \neq \beta} U_{\alpha \beta}\left(n_{\alpha}-\left\langle n_{\alpha}\right\rangle\right)\left(n_{\beta}-\left\langle n_{\beta}\right\rangle\right) .
\end{aligned}
$$

The mean-field Hamiltonian derived from the above Hamiltonian is

$$
\hat{h}=\sum_{\alpha} \tilde{\epsilon}_{\alpha} n_{\alpha}+\sum_{\alpha \neq \beta} t_{\alpha \beta} c_{\alpha}^{\dagger} c_{\beta}+U_{\alpha \alpha}^{\mathrm{SCF}}
$$

where

$$
U_{\alpha \alpha}^{\mathrm{SCF}}=U_{\alpha \alpha}\left(n_{\alpha}-\frac{\left\langle n_{\alpha}\right\rangle}{2}\right)+\frac{1}{2} \sum_{\alpha \neq \beta} U_{\alpha \beta}\left(n_{\beta}-\left\langle n_{\beta}\right\rangle\right) .
$$

is the SCF, the calculation of $\left\langle n_{\alpha}\right\rangle$ performed self-consistently with the one electron Hamiltonian $\hat{h}$. The summation over both indexes $\alpha, \beta$ is performed where indicated. In the following sections, we derive appropriate parameters $\tilde{\epsilon}, t$ and $U$ for benzene, to describe the two different approaches i.e., the CI ((3)) and the SCF approaches ((5)), and compare them in parallel in the case of both equilibrium and nonequilibrium conditions.

Fig. 2(a) shows the selection of mean field on-site energies $\tilde{\epsilon}_{\alpha}$ and hopping parameter $t_{\alpha \beta}$, by comparing the eigen-energies of our model SCF Hamiltonian ((4)) with the frontier orbitals within the local density approximation (LDA) in the 6-31g basis set, shown in the left and right sections of Fig. 2(a) respectively. The carbon-carbon hopping term $t_{\alpha \beta}=-2.0 \mathrm{eV}$ has been used from already tabulated data [20], [41], which yields $\tilde{\epsilon}_{\alpha}=-4.42 \mathrm{eV}$ for the above fit. Note that the highest occupied molecular orbital (HOMO) levels and lowest unoccupied molecular orbital (LUMO) levels are doubly degenerate in our model tight binding Hamiltonian as well as in the LDA basis set.

\section{A. Equilibrium Electron Number Versus Chemical Potential: Choosing Charging Parameters}

A distinguishing aspect of $\mathrm{CB}$ is the abrupt charge addition as opposed to a gradual one in an SCF calculation shown in Fig. 2(b). This fact is readily seen in the figure, in which the 

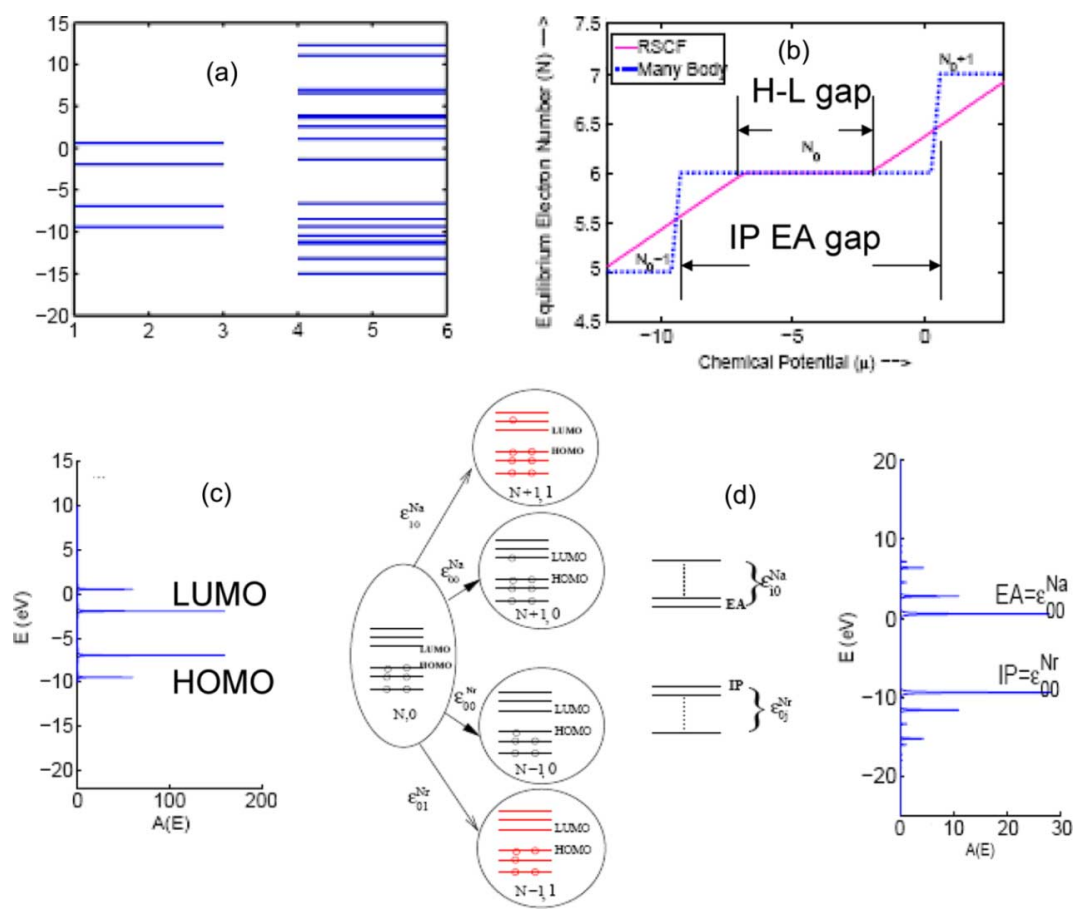

Fig. 2. Model Hamiltonian and equilibrium properties. (a) Selection of on-site $\epsilon_{\alpha}$ and hopping parameter $t_{\alpha \beta}$. Comparison of our model Hamiltonian levels with frontier LDA/6-31g levels. Parameters are fixed based on a close match between the doubly degenerate HOMO and LUMO levels and singly degenerate HOMO-1, LUMO+1 levels. (b) Charging parameter matched according to a consistent restricted SCF-based $N-\mu$ plot (shown continuous line). Total energy based many-body calculation (shown dotted line) as well as RSCF calculation is consistent with Gaussian based calculation [26]. (c) One particle spectral function shows peaks at the energy levels of the single-particle Hamiltonian $\tilde{h}$. (d) Lehmann spectral function evaluated via many-electron spectrum yields many more spectral peaks corresponging to removal (addition) of electrons from the neutral ground state into various charge configurations (excitations) of singly charged species. Notice that the IP-EA and HOMO-LUMO gaps are equal to the corresponding charge-stability plateaus $N=N_{0}$ for many-body and SCF calculations shown in (b).

SCF and CB calculations are presented using the one electron and many-electron Hamiltonians described by (3) and (4) respectively. In the weak coupling limit, the $\mathrm{CB}$ result is more physical, and the SCF calculation does not do justice to this integer charge transfer. However, schemes such as self-interaction correction could be introduced within the one-electron Hamiltonian [21]-[23] to incorporate this. But it turns out that even such schemes may not capture nonequilibrium correctly [9]. It is, however, expected that as coupling strength to the electrodes is increased, the electron transfer resembles the SCF result. There is as yet no clear formalism that addresses [24] this crossover, even in the equilibrium case although the two opposite limits namely SCF and CB are well understood. While the two limits can individually be handled by perturbative expansion in the small parameters $U / \Gamma$ and $\Gamma / U, U$ being the single-electron charging energy and $\Gamma$ being the level broadening, the intermediate regime is hard to handle owing to the nonexistence of a suitable small parameter or "fine structure constant" for transport.

The plateau in the charge addition diagram $N$ versus $\mu$, in which the electron number is stabilized, spans the HOMO-LUMO gap in the SCF case and the ionization potential-electron affinity (IP-EA gap) in the CB calculation. The IP (EA) is defined as the energy when an electron can be removed (added) to the neutral molecule carrying $N_{0}$ electrons. This occurs when the chemical potential $\mu$ equals the energy difference between ground states differing by an electron number $\mu=E_{G}^{N_{0}}-E_{G}^{N_{0}-1}$ for IP, and $\mu=E_{G}^{N_{0}+1}-E_{G}^{N_{0}}$ for EA.
The situation is, however, different in the case of SCF. Here the charge transfer dictated by a self-consistent potential (5) is gradual, in which two electrons are transferred adiabatically over a span of $2 U$ corresponding to the removal of two electrons. This is usually referred to as the restricted SCF (RSCF mentioned in Fig. 2(b)). Most SCF calculations in the literature [25] employ different variants of this scheme. There are also spin unrestricted SCF techniques [21]-[23] which take into account the abrupt charge transfer in a weakly coupled system, due to self-interaction correction, but it is not yet clear whether they work out of equilibrium [9], [27].

One expects that the IP occurs roughly midway during the gradual charge removal in the RSCF scheme [24], [25]. We use this fact to estimate our charging parameters $U_{\alpha \beta}$, with the aid of a Gaussian-98 based calculation for the equilibrium electron number v/s chemical potential $(N-\mu)$, published elsewhere [26]. The calculation corresponding to the equilibrium number of $N_{0}=42$ maps onto our model calculations for $N_{0}=6$, focussing thus on the frontier orbitals and ignoring the inner core that is frozen in our estimate for $\tilde{\epsilon}_{\alpha}$. By implementing a restricted SCF scheme using (5) within in our model Hamiltonian, we obtain a close match of the $N-\mu$ plots in the range between $N_{0}$ and $N_{0}-1$ in comparison with the Gaussian-98 calculation in [26]. Using an estimate of the onsite charging $U_{\alpha \alpha}$, we calculate $U_{\alpha \beta}$ using the Matago-Nishimoto approximation

$$
U_{\alpha \beta}=\frac{e^{2}}{4 \pi \epsilon_{0} r_{\alpha \beta}+\frac{2 e^{2}}{U_{\alpha \alpha}+U_{\beta \beta}}}
$$


where $r_{\alpha \beta}$ is the intercarbon distance in benzene. In each case, evaluation of $n_{\alpha}$ is done self-consistently using an equilibrium value $N_{0}=6$, and $\left\langle n_{\alpha \sigma}\right\rangle=1 / 2$. Using exact eigen-energies of the many-electron Hamiltonian (3) with the above parameters, an $N-\mu$ calculation using these total energies (shown dotted red in Fig. 2(b)) is in excellent agreement with respect to Gaussian calculations in [26]. Note that the $\mu=I P$ in Fig. 2(b) occurs midway between $N=N_{0}$ and $N=N_{0}-2$ in the RSCF charging diagram. It is worth mentioning that the many-body calculation presented in this figure takes all correlation energies into account and is the exact ground state energy within our defined model Hamiltonian.

\section{B. Equilibrium Spectral Function}

Conduction through molecules via molecular orbitals is well understood in the SCF picture [28]. In the strongly coupled regime (most appropriate for an SCF treatment), fractional charge transfer occurs, and density of states (DOS) is evaluated at equilibrium [1] in order to capture the effect of the strong coupling with contact. An interplay of molecular DOS and charging treated self-consistently determines the nonequilibrium response (current-voltage or $I-V$ characteristics). The density of states calculated from the one-electron Green's function [1] in Fig. 2(c) shows peaks at the single-electron eigen spectrum. As the coupling to electrodes gets stronger, the single-electron DOS will show signatures and artifacts of contact bondings [15], [28].

In the weak coupling (CB) limit, however, integer charge addition is favored, and transitions between states that differ by a single electron appear as spectral signatures [19], [40]. At equilibrium, it is convenient to introduce the Ground State Spectral Function by defining the Green's function in the Lehmann representation [25]

$$
\begin{aligned}
G_{\alpha \beta}(E)= & \frac{\left\langle N, 0\left|c_{\alpha}\right| N+1, j\right\rangle\left\langle N+1, j\left|c_{\beta}^{\dagger}\right| N, 0\right\rangle}{E+i 0^{+}-\left(E_{j}^{N+1}-E_{0}^{N}\right)} \\
& +\frac{\left\langle N, 0\left|c_{\beta}^{\dagger}\right| N-1, j\right\rangle\left\langle N-1, j\left|c_{\alpha}\right| N, 0\right\rangle}{E+i 0^{+}-\left(E_{0}^{N}-E_{j}^{N-1}\right)} \\
A_{\alpha \beta}(E)= & i\left[G(E)-G^{\dagger}(E)\right]
\end{aligned}
$$

where $\alpha, \beta$ correspond to the orbital index, which in our case are the sites of the benzene molecule, and $|N, i\rangle$ denotes the $i^{\text {th }}$ excited state of a charge configuration of $N$ electrons. The poles of this spectral function represent various transition energies for addition (removal) of electrons from the neutral ground state

$$
\begin{aligned}
& \epsilon_{0 j}^{N r}=E_{0}^{N}-E_{j}^{N-1} \\
& \epsilon_{0 j}^{N a}=E_{j}^{N+1}-E_{0}^{N}
\end{aligned}
$$

whose spectral strengths are given by

$$
\begin{aligned}
& \tau_{0 j, \alpha \beta}^{N r}=\left\langle N, 0\left|c_{\alpha}\right| N+1, j\right\rangle\left\langle N+1, j\left|c_{\beta}^{\dagger}\right| N, 0\right\rangle \\
& \tau_{0 j, \alpha \beta}^{N a}=\left\langle N, 0\left|c_{\beta}^{\dagger}\right| N-1, j\right\rangle\left\langle N-1, j\left|c_{\alpha}\right| N, 0\right\rangle
\end{aligned}
$$

The first (addition) term adds an electron to orbital $\beta$, taking the system from an $N$ electron ground state to the $j^{\text {th }}(N+$ 1) electron excited state, and then removes it from orbital $\alpha$, bringing it back to ground state. The second (removal) equation first removes an electron from $\alpha$ and then adds it to $\beta$.

One can rewrite the expression in terms of diagonal terms only, replacing the recurring index $\alpha$ with a single index, in a more convenient form as

$$
A_{0 \alpha}^{N}(E)=\sum_{j}\left[\tau_{0 j \alpha}^{N r} \delta\left(E-\epsilon_{0 j}^{N r}\right)+\tau_{0 j \alpha}^{N a} \delta\left(E-\epsilon_{0 j}^{N a}\right)\right]
$$

The spectral function shown in Fig. 2(d) represents the removal and addition strength of various transitions at their energies given by (10). Notice that there are numerous peaks in this spectrum calculated from the many-electron transitions, due to the possible transfer to various excited states of charged species shown in Fig. 2(d). It is important to note that although each transition has a nontrivial spectral weight given by (9), they satisfy an overall sum rule that amounts to the total electron number in the system. We will see in subsequent sections that these transitions involving excited states show up directly as transport signatures frequently observed in experiments.

\section{NONEQUILIBRIUM}

This section is devoted to the various unique transport signatures in the weak coupling (CB) regime, many of which have experimental significance. We elaborate on how various excited states get accessed as a result of contacts maintained at different potentials (nonequilibrium), and what the SCF theory completely misses in this regime. Throughout this paper we describe the electrodes (contacts) using corresponding electrochemical potentials $\mu_{L}$ and $\mu_{R}$ and coupling strengths $\gamma_{L}$ and $\gamma_{R}$.

\section{A. Coulomb Blockade Approach: Rate Equation Model}

Transport in the CB limit [17], [19], [29], [40] is often modeled with a rate equation approach, in which the steady state addition and removal of electrons is described with a rate equation for the nonequilibrium probability $P_{i}^{N}$ of each $N$ electron many-body state $|N, i\rangle$ with total energy $E_{i}^{N}$. The master equation involves transition rates $R_{(N, i) \rightarrow(N \pm 1, j)}$ between states differing by a single electron, leading to a set of independent equations defined by the size of the Fock space [19], [40]

$$
\frac{d P_{i}^{N}}{d t}=-\sum_{j}\left[R_{(N, i) \rightarrow(N \pm 1, j)} P_{i}^{N}-R_{(N \pm 1, j) \rightarrow(N, i)} P_{j}^{N \pm 1}\right]
$$

along with the normalization equation $\sum_{i, N} P_{i}^{N}=1$. We define rate constants

$$
\begin{aligned}
& \Gamma_{i j \alpha}^{N r}=\gamma_{\alpha}\left|\tau_{i j \alpha}^{N r}\right|^{2} \\
& \Gamma_{i j \alpha}^{N a}=\gamma_{\alpha}\left|\tau_{i j \alpha}^{N a}\right|^{2}
\end{aligned}
$$

where $\gamma_{\alpha}$ represents lead molecule broadening or coupling via the end atoms, described using Fermi's golden rule. These constants represent the partial probability for the electron to be in- 
jected by the end atom into a given many-electron ground or excited state. The transition rates are now given by

$$
\begin{aligned}
& R_{(N, i) \rightarrow(N-1, j)}=\sum_{\alpha=L, R} \Gamma_{i j \alpha}^{N r}\left[1-f\left(\epsilon_{i j}^{N r}-\mu_{\alpha}\right)\right] \\
& R_{(N-1, j) \rightarrow(N, i)}=\sum_{\alpha=L, R} \Gamma_{i j \alpha}^{N r} f\left(\epsilon_{i j}^{N r}-\mu_{\alpha}\right)
\end{aligned}
$$

for the removal levels $(N, i \leftrightarrow N-1, j)$, and replacing $(r \rightarrow$ $a, f \rightarrow 1-f)$ for the addition levels $(N, i \leftrightarrow N+1, j) . \mu_{\alpha}$ are the contact electrochemical potentials, $f$ is the corresponding Fermi function, with single-particle removal and addition energies $\epsilon_{i j}^{N r}=E_{i}^{N}-E_{j}^{N-1}$, and $\epsilon_{i j}^{N a}=E_{j}^{N+1}-E_{i}^{N}$. Finally, the steady-state solution to ((11)) is used to get the left terminal current as

$$
I= \pm \frac{e}{\hbar} \sum_{i j}\left[R_{(N, i) \rightarrow(N \pm 1, j)}^{L} P_{i}^{N}-R_{(N \pm 1, j) \rightarrow(N, i)}^{L} P_{j}^{N \pm 1}\right]
$$

where states corresponding to a removal of electrons by the left electrode involve a negative sign.

\section{B. STM Limit}

We briefly elucidate the relationship between spectral functions defined earlier and STM conduction spectra. Electrical conduction depends on the measurement geometry [28] and charging determined by capacitive voltage-division ratio $\eta$ between leads in the Laplace solution as opposed to the resistive voltage-division ratio $\gamma=\gamma_{R} / \gamma_{L}$ which determines the extent to which the levels are filled or emptied by the leads. The source and drain potentials are then given by $\mu_{L}=E_{F}+\eta V_{d}$ and $\mu_{R}=E_{F}-(1-\eta) V_{d}$.

Consider a simple picture in which one contact is very weakly coupled $(\gamma \ll 1, \eta=0$ ), equivalent to the molecule being in equilibrium with left contact $\mu_{L}$. In the $\eta=0$ limit the molecular energy levels are pinned to this contact implying that for a positive voltage $\mu_{R}<\mu_{L}$, and $\mu_{L}$ remains at the equilibrium position. This picture is analogous to STM shell tunneling experiments [32], [42], in which the weakly coupled STM tip acts as a voltage probe, thereby generating the single-particle spectrum, the molecule/dot held in equilibrium with the more strongly coupled contact, in this case, the substrate.

- Ground state spectral function: It is expected, with a more strongly coupled contact, that the right contact voltage probe, such as an STM tip can add or withdraw into or out of the dot at energies corresponding to addition or removal energies defined in (17). The stronger coupling to the left contact ensures that an electron be added or removed as soon as the tip removes or adds an electron thus maintaining overall charge neutrality. In this case conductance spectrum proportional to the equilibrium spectral function is obtained as shown in Fig. 3(b).

- Excited spectral functions: In the previous case, the chemical potential of the left contact is fixed above the transition level $\epsilon_{00}^{N r}$ but below $\epsilon_{10}^{N r}$, thus maintaining the molecule's charge neutrality in its ground state (i.e., $|N, 0\rangle$ ), and hence only the ground state spectral signature $A_{0, j}^{N}(E)$ is observed. However, in a general nonequilibrium scenario, access to excited states of the neutral and charged molecule
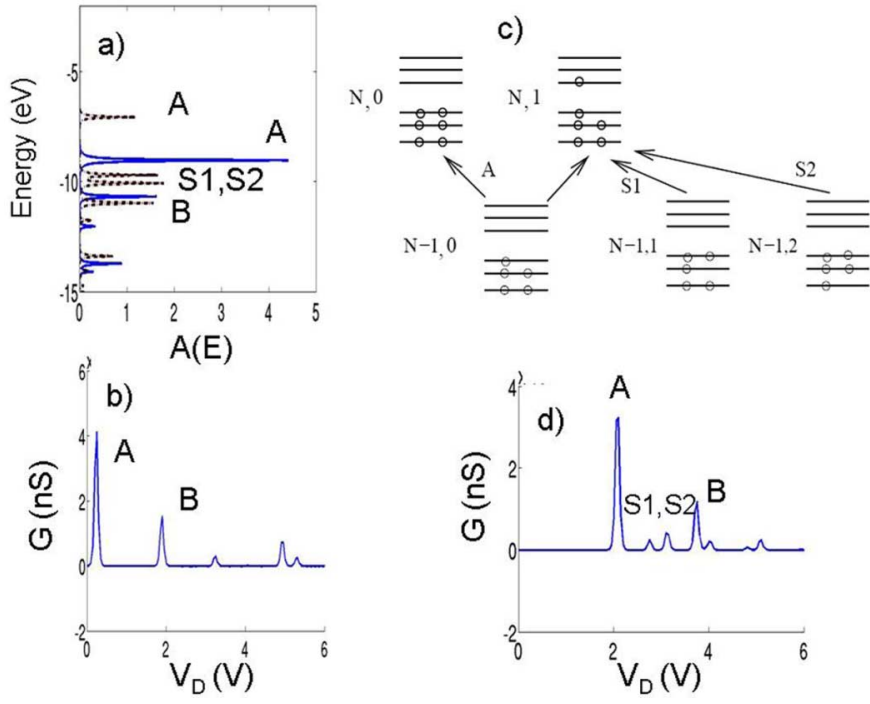

Fig. 3. STM limit-mapping spectral signatures. (a) Removal spectral functions of ground state $A_{0, L / R}^{N r}(E)$ (continuous) and first excited state $A_{1, \alpha}^{N}(E)$ reproduced in the STM spectra in (b). The STM spectra can also show signatures of charge neutral excited states shown dotted in (a), depending on the position of the equilibrium chemical potential (see text). (c) Simple schematic depicting the interplay of $A_{0, L / R}^{N r}(E)$ and $A_{1, L / R}^{N}(E)$ resulting in satellite peaks $S 1$ and $\mathrm{S} 2$ in conduction spectra of lower right half. (d) Conductance spectra reproducing features of both ground and excited state spectral functions $A_{0, L / R}^{N r}(E)$ and $A_{1, \alpha}^{N}(E)$.

becomes feasible and hence description in terms of spectral functions corresponding to addition/removal from the $i^{t h}$ excited state of the neutral molecule is required

$A_{i, \alpha}^{N}(E)=\sum_{j}\left[\tau_{i j \alpha}^{N r} \delta\left(E-\epsilon_{i j}^{N r}\right)+\tau_{i j \alpha}^{N a} \delta\left(E-\epsilon_{i j}^{N a}\right)\right]$

where $\alpha$ now corresponds to the two sites that are coupled to the left (L)/right (R) contacts. For example, let the equilibrium chemical potential be situated at a position above $\epsilon_{10}^{N r}=E_{1}^{N}-E_{0}^{N-1}$, shown dotted in Fig. 3(a). Given, a positive bias $\left(\mu_{L}>\mu_{R}\right)$ the above transition is energetically feasible only if the ground state of the cation $(|N-1,0\rangle)$ is accessed, which occurs for a tip voltage corresponding to $\mu_{R}$ below $\epsilon_{00}^{N r}$. Once this transition is accessed, spectral function $A_{1, L / R}^{N}$ involving the first excited state gets involved due to the initial condiction $\mu_{L}>\epsilon_{10}^{N r}$, due to which the neutral excited state $|N, 1\rangle$ can be accessed. This results in additional satellite peaks S1 and S2 in Fig. 3(d). A schematic of transitions that consititute the satellite peaks $\mathrm{S} 1$ and $\mathrm{S} 2$ due to $A_{1, \alpha}^{N}(E)$ is shown in Fig. 3(c). In this figure, we have only shown the removal levels for brevity, and extension of the argument by including addition levels is trivial. In general, in the STM regime, one can write a simple expression to evaluate the conductance formula as a weighted average over various excited state spectral functions

$$
\frac{d I}{d V_{R}} \approx \frac{e^{2}}{h} \gamma_{R} \sum_{i} P_{i}^{N} A_{i R}^{N}\left(\mu_{R}\right) .
$$

We have thus shown a simple signature that indicates the access of excitations in the many-body spectrum of the neutral mole- 


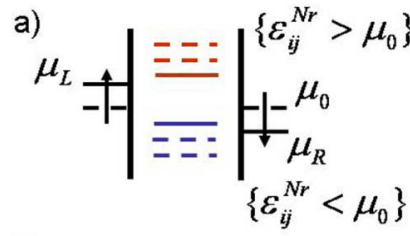

b)
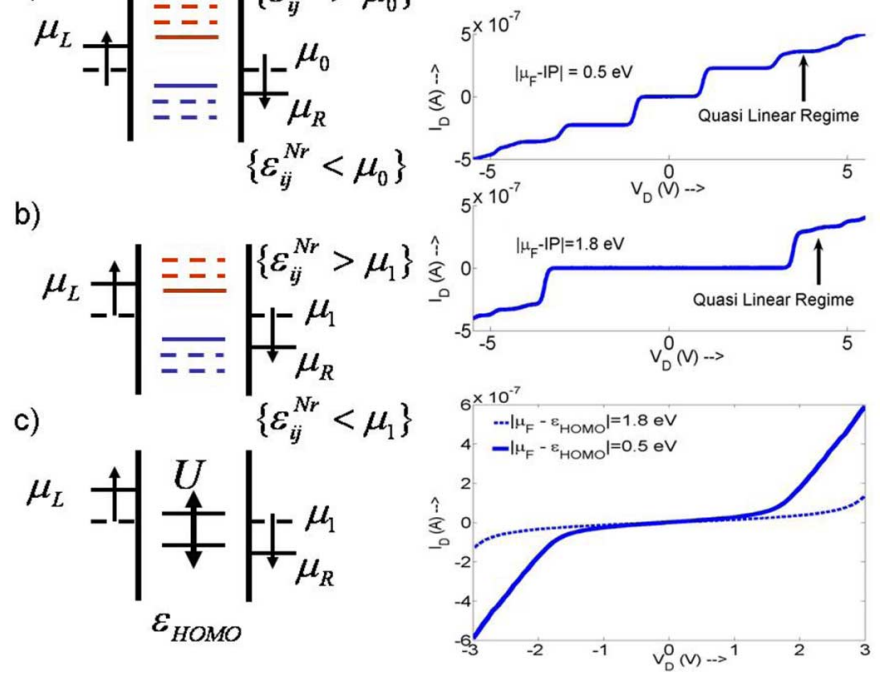

Fig. 4. CB transport under identical contact coupling. (a) Schematic of CB conduction resulting in qualitatively different $I-V$ characteristics. For 1) $\mu_{F}=$ $\mu_{0}$ one observes $I-V$ with a coulomb staircase with a plateau followed by a quasi-linear rise. 2) $\mu_{F}=\mu_{1}$ one observes the quasi-linear $I-V$ upon reaching threshold. (b) This occurs with the intersection of $\mu_{L}=\epsilon_{10}^{N r}$ and $\mu_{R}=$ $\epsilon_{00}^{N r}$ line in the stability diagram. Stability diagram shown for $N=6$ particle blockade region. (c) Distinct $I-V$ 's under cross sections $\mu_{F}=\mu_{0}$ and $\mu_{F}=\mu_{1}$.

cule. In general, one may view near-equilibrium conduction in the $\mathrm{CB}$ regime using single-particle energy levels

$$
\epsilon_{i j}^{N}=E_{i}^{N}-E_{j}^{N-1}
$$

and their corresponding spectral weights.

\section{Break Junction Limit}

The break junction limit is achieved by setting $\eta=0.5$, $\gamma=1$, implying that both contacts are equally coupled to the molecular dot and half the applied voltage appears across the molecular levels which in our case transition energies $\epsilon_{i j}^{N}$. The many-body configuration of the molecule consists of its ground state $|N, 0\rangle$ and the first excited state $|N, 1\rangle$ separated by a gap similar to the HOMO-LUMO gap $\Delta$, followed by a set of closely spaced excitations denoted by $|N, i\rangle, i>1$. The $I-V$ characteristics in this limit show certain key signatures which result from how these excitations are accessed.

The onset of conduction is established by the offset between the equilibrium Fermi energy $E_{F}$ and the first accessible transition energy $\epsilon_{00}^{N r}$. The qualitative shape of the $I-V$ 's depends on how the excitations are accessed. Recall that $\eta=0.5$ implies that the molecular levels are displaced with respect to the contact electrochemical potentials by the applied voltage. If the excited states are not accessed simultaneously or prior to the threshold transition $\epsilon_{00}^{N r}$, as shown in Fig. 4(b), the $I-V$ has a brief staircase of plateaus before a quasi-linear rise in current. This quasi-linear current rise occurs due to a huge number of closely spaced transport channels that are triggered only when transitions involving an excitation appear within the bias window. However, the quasi-linear current can also appear prematurely without an intervening plateau, if a feasible transition to an excited state appears in the bias window at or before the threshold transition.
This situation is shown in Fig. 4(b), where $\epsilon_{10}^{N r}$ also appears at threshold, resulting in a quasi-linear regime immediately following the onset. The two distinct $I-V$ 's have been observed experimentally [10]-[13], [31] and depend merely on the position of the equilibrium electrochemical potential (Fermi energy) with respect to the transition energies. In the meanwhile, similar SCF-based $I-V$ characteristics show adiabatically smeared out currents whose onsets get postponed by the changing position of the equilibrium $E_{F}$, as shown in Fig. 4(c). The SCF potential from (5) determines how levels float with respect to their nonequilibrium occupation [1]. It is readily seen by comparing Fig. 3(a) and (b) with Fig. 3(c) that any self-consistent potential cannot change the qualitative features of the $I-V^{\prime}$ 's in order to resemble the $\mathrm{CB}$ features.

\section{Connection to Experiments: Fitting Data Using Coulomb Blockade Model}

We consider matching the $I-V$ shapes using our CB model using consistent fitting parameters. The experiments conducted on conjugated phenylenes [11], [12] at low temperatures $(T=$ $30 \mathrm{~K}$ ) suggest strong $\mathrm{CB}$ effects. It is worth noting that an "orthodox" theory simply involving junction resistors and capacitors would also manage to capture the zero-bias suppressed conductance, the subsequent sharp onset and the linear current rise; however, it would not capture the intervening plateaus, fine structures in the $I-V$ 's, and some asymmetry features that arise due to discrete transitions in the molecular configuration space [10], [14]. In contrast with metallic islands, a molecular dot shows significant size quantization that leads to quantum corrections to the junction capacitance, and gets further modified at high bias to involve nonlinear corrections to it arising from partial densities of states filled separately by the two contacts.

While our model explains salient features of a lot of CB experiments [10], [14], [31] it is interesting to note that in some cases the same molecule showed CB behavior at low temperature and SCF behavior at higher temperatures [11], [12]. A possible explanation is that at low temperature the molecule could be frozen into a configuration where the plane of the middle ring is oriented perpendicular to the side rings, while room temperature structures sample other configurations and are rotated on average. This is supported by the fact that current levels at room temperature are an order of magnitude greater, which can be attributed to an increased average degree of conjugation along the molecular backbone. In contrast at low temperature the rotated central ring has a weaker coupling with the rest of the backbone, which could reduce its broadening while increasing electron localization and charging, leading to $\mathrm{CB}$ behavior. In fact some of the experiments feature bulky middle groups like antracence. Steric side groups that are deliberately inserted to facilitate this rotation of the central rings and enforce $\mathrm{CB}$ [12]. While doing exact calculations on these molecular structures is beyond the scope of the present paper, we consider making simple fits by considering the following facts.

- Current levels: Using the fitting parameters $\gamma_{1}=\gamma_{2} \approx$ 5-10 meV we obtain current levels similar to experimental data. It is important to note that changing $\gamma$ does not affect the conductance before the threshold voltage which shows a vanishingly small prethreshold current. 


\section{THEORY}
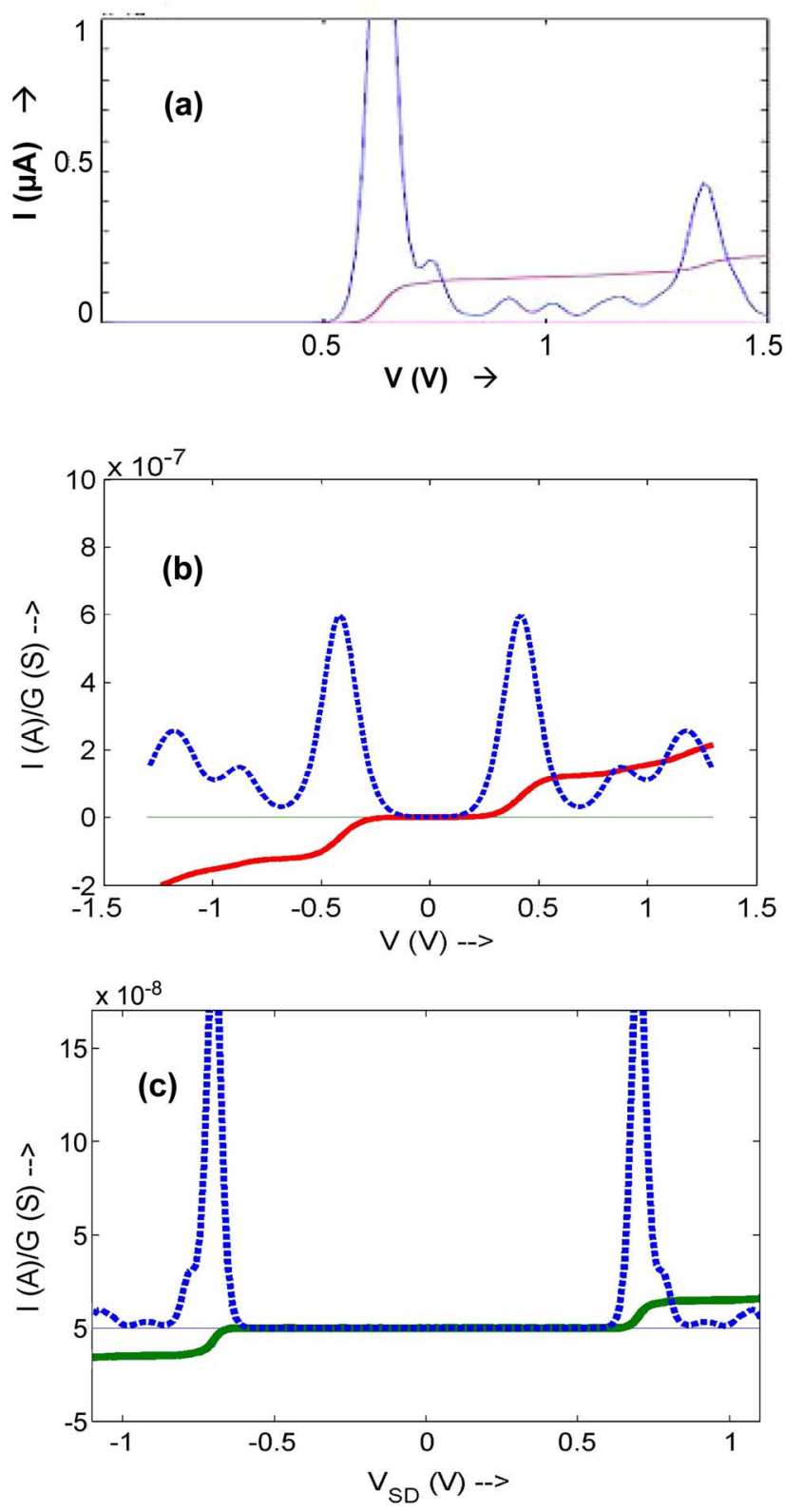

EXPERIMENT
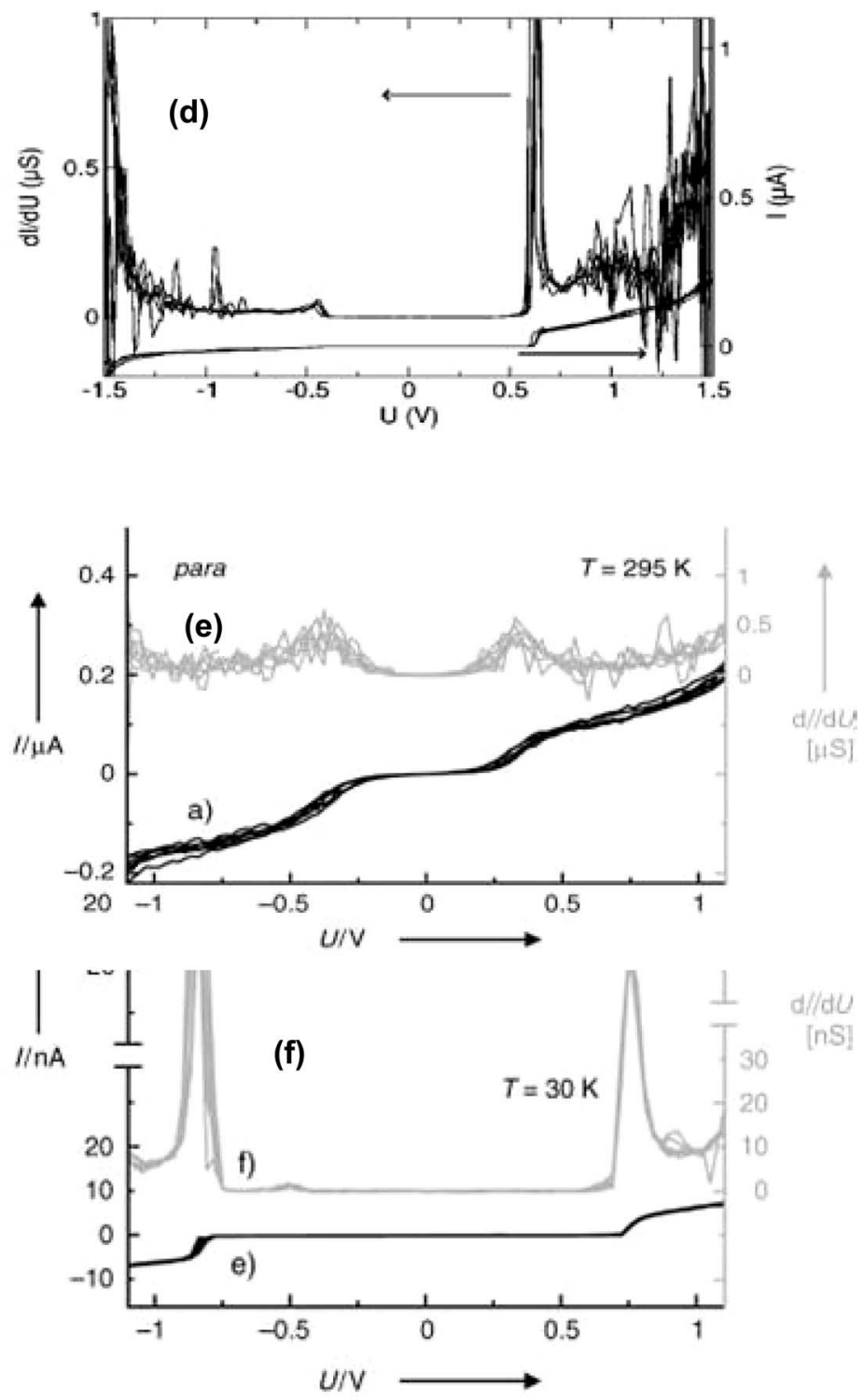

Fig. 5. Experimental fits for data [11], [12]. (a) $T=30 \mathrm{~K}, \gamma \approx 5 \mathrm{meV}$. (b) $T=295 \mathrm{~K}, \gamma \approx 5 \mathrm{meV}$. (c) $T=30 \mathrm{~K}, \gamma \approx 0.25 \mathrm{meV}$.

- Threshold voltage: We noticed in the last section with that the gap $\Delta$ between ground and first excited states of the neutral molecule is important in determining the qualitative shape of the $I-V$. When the equilibrium electrochemical potential $E_{F}$ lies above midgap between $\epsilon_{10}^{N r}$ and $\epsilon_{00}^{N r}$, the first excited state becomes voltage-accessible before the ground state of the charged species is accessed and populated simultaneously via $\mu_{R}=\epsilon_{00}^{N r}$, giving rise to the quasi- linear $I-V$ immediately following the very first current onset. In all the experimental data, we observe a threshold voltage between 0.5 and $0.7 \mathrm{~V}$ thus tuning the gap $\Delta \approx 0.6-0.8 \mathrm{~V}$.

Fig. 5(a), (b), and (c) are fits obtained for experiments [11], [12]. In case of molecular asymmetries [11], only positive bias is considered [9], the $I-V$ asymmetries themselves being attributed to polarization effects [7]. In obtaining an experimental fit for [12], in Fig. 5(b), we used $T=295 \mathrm{~K}$ consistent with experiment. Notice that the first peak has broadened significantly. The higher temperature coulomb blockade could possibly be attibuted to the fact that molecule involved has an anthracene based middle ring that is much bulkier, thus leading to a higher temperature frozen configuration stabilized by steric interactions.

The molecular system we consider is a simple prototypical molecule (benzene in our case) with calculations based on simple parameters that are associated with this minimal system. Performing calculations on a real molecule-electrode system will be needed to yield a quantitative fit in terms of threshold voltage, current levels and positions of peaks. However, the conduction mechanism remains the same. The exponentially larger configuration space of even a minimal coulomb blockaded molecule makes a first-principles calculation of its transport properties inordinately challenging compared to SCF 
treatments in the literature. However, the SCF calculations do not capture the nonequilibrium transition rates between the many-body states, which as we argued earlier carry crucial correlation signatures that are experimentally observable for ultrashort molecules. Such a "real" calculation involving the quantum chemistry of larger molecules and contact bondings within this nonequilibrium full CI treatment is still at a very early stage [33]. Furthermore, it needs to be supplemented with the broadening of the many-particle states that could affect the interference between nearby levels, an issue that has received relatively little attention [34]-[39] and requires further work.

\section{CONCLUSION}

In this paper, we have developed a $\mathrm{CB}$ approach for molecular conduction through short molecules using benzene as a prototype. We have shown how equilibrium and nonequilibrium signatures are very different from the traditional NEGF-SCF viewpoint, and that the CB approach is appropriate in the weak coupling limit. Many $I-V$ features distinct to the $\mathrm{CB}$ regime are often seen in experiments. These features that are easily obtained using a full CI master equation approach are potentially very hard to obtain within any effective one-electron potential, even for a minimal model. A particular challenge therefore lies in bridging the $\mathrm{SCF}$ and $\mathrm{CB}$ regimes while paying close attention to coherent level broadening and associated interferences. The emergence of many recent experiments on molecular dots, exploring the interplay between charging, quantization, and level-broadening, should prove invaluable in further theoretical developments in this regard.

\section{REFERENCES}

[1] S. Datta, Quantum Transport: Atom to Transistor. Cambridge, U.K.: Cambridge Univ. Press, 2005.

[2] Y. Meir and N. S. Wingreen, "Landauer formula for the current through an interacting electron region," Phys. Rev. Lett, vol. 68, pp. 2512-2515, 1992.

[3] M. D. Ventra, S. T. Pantelides, and N. D. Lang, "First-principles calculation of transport properties of a molecular device," Phys. Rev. Lett., vol. 84, pp. 979-982, 2000.

[4] P. S. Damle, A. W. Ghosh, and S. Datta, "First principles analysis of molecular conduction using quantum chemical software," Chem. Phys., vol. 281, pp. 171-188, 2002.

[5] J. Taylor, H. Guo, and J. Wang, "Ab initio modeling of quantum transport properties of molecular electronic devices," Phys. Rev. B, vol. 63, pp. 245 407-1-245 407-13, 2001.

[6] A. W. Ghosh, P. S. Damle, S. Datta, and A. Nitzan, "Molecular electronics: Theory and prospects," MRS Bull., vol. 29, pp. 391-395, 2004.

[7] F. Zahid, A. W. Ghosh, M. Paulsson, E. Polizzi, and S. Datta, "Charging-induced asymmetry in molecular conductors," Phys. Rev. $B$, vol. 70, pp. 245317-1-245317-5, 2004.

[8] M. A. Reed, C. Zhou, D. J. Muller, T. P. Burgin, and J. M. Tour, "Conductance of a molecular junction," Science, vol. 278, pp. 252-254, 1997.

[9] B. Muralidharan, A. W. Ghosh, and S. Datta, "Probing electronic excitations in molecular conduction," Phys. Rev. B, vol. 73, pp. 155410 1-155 410-5, 2006.

[10] J. Park, A. N. Pasupathy, J. I. Goldsmith, C. Chang, Y. Yaish, J. R. Petta, M. Rinkoski, J. P. Sethna, H. D. Abruna, P. L. McEuen, and D. C. Ralph, "Coulomb blockade and the Kondo effect in single-atom transistors," Nature, vol. 417, pp. 722-725, 2002.

[11] J. Reichert, H. B. Weber, M. Mayor, and H. V. Lohneysen, "Low-temperature Conductance measurements on single molecules," Appl. Phys. Lett., vol. 82, pp. 4137-4139, 2003.
[12] M. Mayor, H. B. Weber, J. Reichert, M. Elbing, C. von Hanisch, D. Beckmann, and M. Fischer, "Electric current through a molecular rod-Relevance of the position of the anchor groups," Angew. Chemie Int. Ed., vol. 42, pp. 5834-5838, 2003.

[13] M. Elbing, R. Ochs, M. Koentopp, M. Fischer, C. von Hanisch, F. Evers, H. B. Weber, and M. Mayor, "A single-molecule diode," Proc. Nat. Acad. Sci., vol. 102, pp. 8815-8820, 2005.

[14] G. D. Scott, K. S. Chichak, A. J. Peters, S. J. Cantrill, J. F. Stoddart, and H.-W. Jiang, "Differential conductance peak exchange in Borromean ring single molecule transistors," Condens. Matter, pp. 1-7, Apr. 2005.

[15] G.-C. Liang and A. W. Ghosh, "Identifying contact effects in electronic conduction through C60 on silicon," Phys. Rev. Lett., vol. 95, pp. 076 403-1-076 403-4, 2005.

[16] A. Szabo and N. S. Ostlund, Modern Quantum Chemistry: Introduction to Advanced Electronic Structure Theory. Mineola, NY: Dover, 1996.

[17] M. H. Hettler, W. Wenzel, M. R. Wagewijs, and H. Schoeller, "Current collapse in tunneling transport through benzene," Phys. Rev. Lett., vol. 90, pp. 076 805-1-076805-4, 2003.

[18] L. A. Siddiqui, A. W. Ghosh, and S. Datta, "Phonon runaway in carbon nanotube quantum dots," Phys. Rev. B, vol. 76, pp. 085 433-1-085 433-9, 2007.

[19] E. Bonet, M. M. Deshmukh, and D. C. Ralph, "Solving rate equations for electron tunneling via discrete quantum states," Phys. Rev. B, vol. 65, pp. 045 317-1-045 317-10, 2002.

[20] G.-C. Liang, A. W. Ghosh, M. Paulsson, and S Datta, "Electrostatic potential profiles of molecular conductors," Phys. Rev. B, vol. 69, pp. $115302-1-115302-12,2004$

[21] J. J. Palacios, "Coulomb blockade in electron transport through a C60 molecule from first principles," Phys. Rev. B, vol. 72, pp. 125424 $1-125424-6,2005$.

[22] C. Toher, A. Fillipetti, S. Sanvito, and K. Burke, "Self-interaction errors in density-functional calculations of electronic transport," Phys. Rev. Lett, vol. 95, pp. 146 402-1-146402-4, 2005.

[23] P. Pals and A. MacKinnon, "Coherent tunnelling through two quantum dots with Coulomb interaction," J. Phys., Condens. Matter., vol. 8, pp. 5401-5414, 1996.

[24] K. Matveev, "Coulomb blockade at almost perfect transmission," Phys. Rev. B., vol. 51, pp. 1743-1751, 1995.

[25] P. Fulde, Electron Correlations in Molecules and Solids, ser Springer Series in Solid-State Sciences 100. Berlin, Germany: Springer-Verlag, 1991.

[26] T. Rakshit and S. Datta, unpublished.

[27] B. Muralidharan, A. W. Ghosh, and S. Datta, "Conductance in Coulomb blockaded molecules-Fingerprints of wave-particle duality?," J. Mol. Simul., pp. 751-758, 2006.

[28] F. Zahid, M. Paulsson, and S. Datta, "Electrical conduction through molecules," in Advanced Semiconductors and Organic Nanotechniques, H. Morkoc, Ed. New York: Academic, 2003.

[29] Single Charge Tunneling, ser. NATO ASI series 294, H. Grabert and M. H. Devoret, Eds. New York: Plenum, 1992.

[30] M. M. Deshmukh, E. Bonet, A. N. Pasupathy, and D. C. Ralph, "Equilibrium and nonequilibrium electron tunneling via discrete quantum states," Phys. Rev. B, vol. 65, pp. 073 301-1-073 301-4, 2002.

[31] J.-O. Lee, G. Lientschnig, F. Wiertz, M. Struijk, R. A. J. Janssen, R. Egberink, D. N. Reinhoudt, P. Hadley, and C. Dekker, "Absence of strong gate effects in electrical measurements on phenylene-based conjugated molecules," Nano Lett., vol. 3, pp. 113-117, 2003.

[32] U. Banin, Y. Cao, D. Katz, and O Millo, "Identification of atomiclike states in InAs nanocrystal quantum dots," Nature, vol. 400, pp. 542-544, 1995.

[33] P. Delaney and J. C. Greer, "Correlated electron transport in molecular electronics," Phys. Rev. Lett, vol. 93, pp. 036 805-1-036 805-4, 2004.

[34] H. Haug and A.-P. Jauho, Quantum Kinetics in Transport and Optics of Semiconductors, ser. Springer Series in Solid-State Sciences 123. Berlin, Germany: Springer-Verlag, 1996.

[35] S. Braig and P. W. Brouwer, "Rate equations for Coulomb blockade with ferromagnetic leads," Phys. Rev. B, vol. 71, pp. 195324-1-195 324-9, 2005.

[36] Y. Meir, N. S. Wingreen, and P. A. Lee, "Transport through a strongly interacting electron system: Theory of periodic conductance oscillations," Phys. Rev. Lett., vol. 66, pp. 3048-3051, 1991.

[37] S. A. Gurvitz and Y. S. Prager, "Microscopic derivation of rate equations for quantum transport," Phys. Rev. B, vol. 53, pp. 15 932-15943, 1996.

[38] J. N. Pedersen and A. Wacker, "Tunneling through nanosystems: Combining broadening with many-particle states," Phys. Rev. B, vol. 72, pp. 195 330-1-195 330-9, 2005. 
[39] S. Datta, "Fock Space formulation for nanoscale transport," cond-mat 0603034 .

[40] C. W. J. Beenakker, "Theory of Coulomb-blockade oscillations in the conductance of a quantum dot," Phys. Rev. B, vol. 44, pp. 1646-1656, 1991.

[41] M. Paulsson and S. Stafstrom, "Self-consistent-field study of conduction through conjugated molecules," Phys. Rev. B, vol. 64, pp. 035416 $1-035416-10,2001$.

[42] E. P. A. M. Bakkers, Z. Hens, A. Zunger, A. Franceschetti, L. P. Kouwenhoven, L. Gurevich, and D. Vanmaekelbergh, "Shell-tunneling spectroscopy of the single-particle energy levels of insulating quantum dots," Nano Lett., vol. 1, pp. 551-556, 2001.

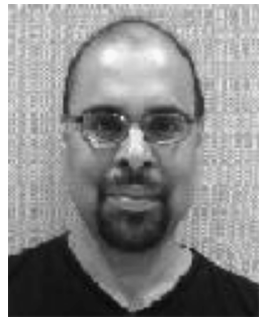

Bhaskaran Muralidharan was born in India. He received the B.Tech. degree in engineering physics from the Indian Institute of Technology, Bombay, in 2001, and the M.S. degree in electrical and computer engineering from Purdue University, West Lafayette, IN, in 2003. He is currently working toward the $\mathrm{Ph} . \mathrm{D}$. degree in electrical and computer engineering.

His current research activity involves Coulomb blockade, quantum dot and molecular transport, and understanding various transport phenomena in the strongly correlated regime.

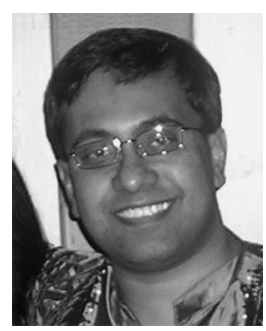

Avik W. Ghosh received the B.S. and M.S. degrees in physics at the Indian Institute of Technology, Kanpur, and the Ph.D. degree in physics in 1999 from Ohio State University, Columbus.

He was employed as a Postdoctoral Fellow and Research Scientist in Electrical Engineering at Purdue University, and is currently an Assistant Professor in Electrical Engineering at the University of Virginia, Charlottesville. His past research activities include ultrafast optical phenomena in semiconductor heterostructures and noise-induced transport in Brownian environments. His current research involves modeling and simulation of electronic conduction in nanoscale devices, including atomic and molecular wires, nanoscale silicon MOSFETs, hybrid silicon-molecular devices, carbon nanotubes, silicon nanowires, and quantum dots.

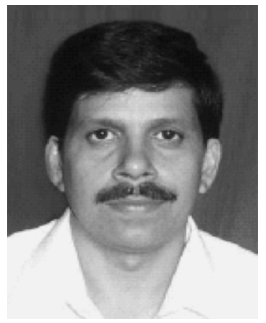

Swapan K. Pati had his early education at the Midnapore College and at Kalyani University, West Bengal. He received the Ph.D. degree from the Indian Institute of Science, Bangalore, followed by postdoctoral work in the department of Physics at University of California, Davis, and in the Chemistry department at Northwestern University.

$\mathrm{He}$ is currently an Associate Professor at the Jawaharlal Nehru Centre for Advanced Scientific Research, Bangalore. He is a Junior Associate of the Abdus Salam International Centre for Theoretical Physics since 2003 and a young affiliate of the Third World Academy of Sciences since 2007. He has also been a Visiting Faculty Member to a number of universities in United States, Europe, and Japan. His research interests include quantum many-body theory, molecular electronics, nonlinear optical phenomena, quantum magnetism and generalized charge transfer mechanisms. $\mathrm{He}$ is also actively involved in developing new theoretical tools for a holistic understanding of structure-property correlations in a whole range of systems from molecules to materials including biological and biomimetic systems (see http://www.jncasr.ac.in/pati/ for more information).

Prof. Pati is a recipient of the bronze medal from the Material Research Society of India (2006) and Chemical Research Society of India (2007).

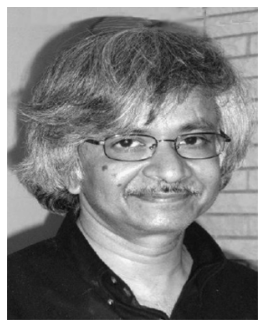

Supriyo Datta (M'82-SM'93-F'96) received the B.Tech. degree from the Indian Institute of Technology, Kharagpur, in 1975 and the Ph.D. degree from the University of Illinois at Urbana-Champaign in 1979.

In 1981, he joined Purdue University, West Lafayette, IN, where he is currently the Thomas Duncan Distinguished Professor in the School of Electrical and Computer Engineering. He has authored several books including Electronic Transport in Mesoscopic Systems (Cambridge, 1995), and Quantum Transport: Atom to Transistor (Cambridge, 2005) and his current research interests are centered on the physics of nanostructures and include spin electronics, molecular electronics, nanoscale device physics, and mesoscopic superconductivity.

Prof. Datta is a Fellow of the American Physical Society (APS) and the Institute of Physics (IOP). In 1984, he received an NSF Presidential Young Investigator Award and an IEEE Centennial Key to the Future Award. In 1994, he was awarded the Frederick Emmons Terman Award from the ASEE. He and his colleague Mark Lundstrom shared the SRC Technical Excellence Award in 2001 and the IEEE Cledo Brunetti Award in 2002. 